\title{
Decreased hyperpolarization-activated cyclic nucleotide-gated channels are involved in bladder dysfunction associated with spinal cord injury
}

\author{
QIAN LIU, CHAO WU, SHENGQUAN HUANG, QINGJIAN WU, TAO ZHOU, \\ XIAOBING LIU, XIN LIU, XIAOYAN HU and LONGKUN LI \\ Department of Urology, The Second Affiliated Hospital, The Third Military Medical University, \\ Chongqing 400037, P.R. China
}

Received November 9, 2016; Accepted February 8, 2018

DOI: $10.3892 / \mathrm{ijmm} .2018 .3489$

\begin{abstract}
Spinal cord injury (SCI) leads to bereft voluntary control of bladder, but the possible role of spontaneous excited system in bladder of SCI patients is poorly understood. Hyperpolarization-activated cyclic nucleotide-gated (HCN) channels are deemed to regulate the spontaneous contraction of bladder, our study explored the functional role of $\mathrm{HCN}$ channels in SCI induced neurogenic bladder. Sixty female Sprague-Dawley rats were randomized into control, sham and SCI groups. Rat models subjected to SCI at S2 levels were successfully established and were assessed using hematoxylin and eosin staining and cystometry. In SCI rats, the mRNA and protein expression levels of HCN channels and the $\mathrm{I}_{\mathrm{h}}$ density were significantly reduced, and expression levels of several bladder HCN1 channel regulatory proteins were also significantly changed. The effects of $50 \mu \mathrm{M}$ forskolin and $50 \mu \mathrm{M} 8$-bromoadenosine 3',5'-cyclic monophosphate on $\left[\mathrm{Ca}^{2+}\right]_{\mathrm{i}}$ of isolated bladder interstitial cells of Cajal-like cells were significantly decreased in SCI rats. The spontaneous contractions in detrusor strips from SCI rats were significantly weakened. Furthermore, detrusor strips from SCI
\end{abstract}

Correspondence to: Professor Longkun Li, Department of Urology, The Second Affiliated Hospital, The Third Military Medical University, Chongqing 400037, P.R. China

E-mail: lilongk@hotmail.com

Abbreviations: SCI, spinal cord injury; HCN, hyperpolarization-activated cyclic nucleotide-gated; FSK, forskolin; 8-Br-cAMP, 8-bromoadenosine 3',5'-cyclic monophosphate; ICC-LCs, interstitial cells of Cajal-like cells; BPS/IC, bladder pain syndrome/interstitial cystitis; DO, detrusor overactive; PBOO, partial bladder outlet obstruction; NRSF, neuronal restrictive silencing factor; Trip $8 \mathrm{~b}$, tetratricopeptide repeat-containing Rab8b-interacting protein; Nedd, neural precursor cell-expressed developmentally downregulated

Key words: hyperpolarization-activated cyclic nucleotide-gated channel, interstitial cells of Cajal-like cells, spontaneous contraction, spinal cord injury, neurogenic bladder rats exhibited decreased tolerance to two doses of ZD7288 (10 and $50 \mu \mathrm{M})$. Taken together, our results indicate that the decreased bladder HCN channel expression and function induced by altered regulatory proteins are involved in the pathological process of SCI induced neurogenic bladder, which present $\mathrm{HCN}$ channels as valid therapeutic targets for treating this disease.

\section{Introduction}

Spinal cord injury (SCI) affects $\sim 2.5$ million people all over the world and there are about 130,000 new cases of SCI each year (1). Body segments below the injury level in SCI patients suffer from chronic paralysis and autonomic dysfunctions, accompanied by several distressful secondary complications, such as spasticity, bowel and bladder dysfunction (2). The bladder dysfunction can result in incontinence and negatively affects the life quality of SCI patients (3). It may also bring about upper urinary tract deterioration and cause the death of SCI patients (4). So far, the therapies for the neurogenic bladder secondary to SCI mainly include catheterization, anticholinergic medications, botulinum toxin A and acupuncture, but no fully restorative treatments have been exploited $(5,6)$. Therefore, more basic scientific research should be performed and try to provide theoretical bases to preferable clinical therapies for the neurogenic bladder due to SCI. Under the premise that it is so difficult to achieve neural regeneration as yet, we should focus on exploring the role of spontaneous excited system in bladder of SCI patients.

Interstitial cells of Cajal (ICCs) were initially identified in 1893 and were demonstrated to act as pacemakers involved in generating slow wave activity and driving peristalsis in the gastrointestinal (GI) tract (7). In recent years, cells resembling the ICC in GI tract have been discovered in the urinary bladder of humans and animals and termed as ICC-like cells (ICC-LCs) (8). ICC-LCs in bladders are deemed to participate in different cellular processes such as pacemaker activity or transmitting neural inputs to detrusor smooth muscle, due to its various locations in the bladder wall (9). Abundant evidence showed that alterations in the distribution and quantity of bladder ICC-LCs were associated with several 
pathological conditions, such as obstructed bladder, bladder pain syndrome/interstitial cystitis (BPS/IC) or diabetic bladder (10). Our previous study suggested that ICC-LCs may be involved in the pathogenesis of SCI induced neurogenic bladder (11). Therefore, the functional role of ICC-LCs in SCI induced neurogenic bladder should be clarified by further research.

Hyperpolarization-activated cyclic nucleotide gated (HCN) channels, which include four subtypes (HCN1-4) in mammals, can generate $I_{h}$ and participate in multiple systemic functions such as heart contractility, hormonal regulation, central pattern generation, sensory perception, learning and memory (12). In a previous study, we detected that all four HCN subtypes are expressed exclusively in bladder ICC-LCs and the HCN1 channel is the prominent one. HCN channels were deemed to be involved in controlling the bladder pacemaker activity via ICC-LCs (13). Abundant evidence suggests that altered $\mathrm{HCN}$ channels are associated with multiple excitatory disorders in heterologous systems, such as various types of epilepsy, sinus bradycardia, atrial fibrillation and Hirschsprung's disease (14-16). Furthermore, we found that HCN channel expression and function in bladder ICC-LCs were significantly increased in detrusor overactive (DO) bladders with partial bladder outlet obstruction (PBOO) (17). However, the role of HCN channels in bladder ICC-LCs of SCI induced neurogenic bladder has not been clarified as yet. In the present study, the altered expression and function, as well as the possible functional roles of HCN channels were investigated in rats with SCI induced neurogenic bladder.

\section{Materials and methods}

Animals. Sixty female Sprague-Dawley rats weighing 150-180 g were used in our study. All the rats were randomly assigned to three groups: control group, sham group and SCI group. All animal experiments were performed according to the Guide for Care and Use of Laboratory Animals issued by the National Institutes of Health, and were authorized by the Research Council and Animal Care and Use Committee of the Third Military Medical University (Chongqing, China).

SCI. Rats were anesthetized by intraperitoneal injection of pentobarbital sodium $(40 \mathrm{mg} / \mathrm{kg})$. Under aseptic conditions, we incised the skin and muscle layers in the rat's back and conducted a laminectomy to expose the spinal cord. Subsequently, we performed complete spinal cord transection at vertebral level S2 in rats, as previously described (18). Rats were subjected to a laminectomy at S2 level but no SCI in sham group and received no treatment in control group. After injury, muscle layers and skin were sutured orderly and rats were injected with $2 \mathrm{ml}$ sterile saline subcutaneously. Then the rats were placed into warmed cages with free access to food and water under a standard 12-h/12-h light/dark cycle. Rats were injected daily with gentamicin subcutaneously $(5 \mathrm{mg} / \mathrm{kg})$ for the first postoperation week. Manual bladder expression was performed twice daily and dehydration was monitored daily. After being maintained for 6 weeks, rats were sacrificed for further research. Three rats in SCI group died of unknown cause before deadline and were excluded from the experiments.
Hematoxylin and eosin (H\&E) staining. The bladders were isolated from the sacrificed rats and fixed with $4 \%$ paraformaldehyde (Boster, Wuhan, China) overnight. Then paraffin-embedded blocks were made and 4- $\mu \mathrm{m}$ sections were manufactured. The H\&E staining was conducted according to a standard protocol (19). Briefly, the sections were first stained in hematoxylin for $30 \mathrm{sec}$ and then 10-30 sec in 1\% eosin solution. Afterwards, the sections were dehydrated in ethanol and fixed with mounting medium. All sections were viewed using a optical microscope (Olympus, Tokyo, Japan).

Urodynamic measurements. As previously described (20), urodynamic measurements were performed in unconscious rats with urethane anesthesia ( $1 \mathrm{~g} / \mathrm{kg}$ body weight, intraperitoneally). PE-50 polyethylene tubing (Becton, Dickinson and Company, Franklin Lakes, NJ, USA) was ducted into the bladder through the urethra. The catheter was connected to a 3-way valve linked with an infusion pump (AVI 270; 3M, Maplewood, MN, USA) and a pressure transducer (Chengyi Co., Chengdu, China), which was calibrated before each experiment. Room temperature saline was infused into the bladder at a constant rate $(10 \mathrm{ml} / \mathrm{h})$ and the pressure transducer output was amplified and visualized using a multi-channel signal processing system (RM6240C; Chengyi Co.).

Western blot analyses. For western blot analysis, rat bladders were lysed in RIPA lysis buffer (Beyotime Institute of Biotechnology, Shanghai, China) to extract total protein and protein concentration was measured using the Bio-Rad DC Protein Assay kit (Bio-Rad Laboratories Inc., Hercules, CA, USA). Protein $(50 \mu \mathrm{g})$ was separated by SDS-PAGE gels and then transferred to PVDF membranes (Merck Millipore, Darmstadt, Germany). Membranes were blocked with 5\% bovine serum albumin in Tris-buffered saline for $2 \mathrm{~h}$ at room temperature and incubated with primary antibodies overnight at $4^{\circ} \mathrm{C}$ as follows: HCN1 (ab84816, 1:1,000), HCN2 (ab65704, 1:1,000), HCN3 (ab84818, 1:1,000) and HCN4 (ab69054, 1:1,000) (all from Abcam, Cambridge, MA, USA), GAPDH (AG019, 1:1,000; Beyotime Institute of Biotechnology), tetratricopeptide repeat-containing Rab8b-interacting protein (Trip8b) (constant, 75-243, 1:800; NeuroMab, Davis, CA, USA), filamin A $(4762,1: 1,000)$ and neural precursor cell-expressed developmentally downregulated (Nedd)4-2 (4013, 1:1,000) (both from Cell Signaling Technology, Inc., Danvers, MA, USA) neuronal restrictive silencing factor (NRSF) (ab21635, 1:1,000; Abcam), tubulin (AT819, 1:1,000; Beyotime Institute of Biotechnology), then primary antibodies was bound by horseradish peroxidase-conjugated secondary antibodies (ZB-2301, ZB-2305, 1:5,000; Zhongshan Golden Bridge Biotechnology Co., Ltd., Beijing, China). Signal was detected using ECL substrate (Millipore, Billerica, MA, USA) and imaged by ImageQuant LAS 4000 Bio-Imaging system (GE Healthcare Life Sciences, Stockholm, Sweden).

Quantitative RT-PCR. Total RNA was extracted from rat bladders using TRIzol reagent (Takara Biotechnology Co., Ltd., Dalian, China) and cDNA was synthesized by PrimeScript ${ }^{\mathrm{TM}}$ RT reagent kit (Takara Biotechnology Co., Ltd.), according to the manufacturer's instructions. Primers used in this study are listed in Table I. Quantitative RT-PCR was performed 
Table I. Primers used for quantitative RT-PCR.

\begin{tabular}{lclr}
\hline Genes & Species & \multicolumn{1}{c}{ Primer sequences } & Accession no. \\
\hline HCN1 & Rat & F: 5'-CATGCCACAGCTTTGATCCAGTCT-3' & NM_053375 \\
HCN2 & Rat & F: 5'-CGCATGTCAGCTGGTAACTTGTG-3' & NM_053684 \\
& R: 5'-CGCCGCATCATGGGGTACTC-3' & \\
HCN3 & Fat & F: 5'-GCAGCGCATCCACGAGTACTACG-3' & NM_053685 \\
HCN4 & Rat & F: 5'-CCCCGGCAGGTGAAGTTAATAATC-3' & NM_021658 \\
GAPDH & R: 5'-CGGGGGTGTCTCTGGTGTACTTG-3' & \\
& Rat & F: 5'-GGCCCCTCTGGAAAGCTGTG-3' & NM_017008
\end{tabular}

F, forward; R, reverse; HCN, hyperpolarization-activated cyclic nucleotide-gated channel; GAPDH, glyceraldehyde 3-phosphate dehydrogenase.

with a StepOnePlus Real-Time PCR system (Invitrogen Life Technologies, Carlsbad, CA, USA) using SYBR-Green Real-time PCR Master mix (Toyobo, Osaka, Japan). The thermocycling program consisted of $95^{\circ} \mathrm{C}$ for $1 \mathrm{~min}, 95^{\circ} \mathrm{C}$ for $15 \mathrm{sec}, 60^{\circ} \mathrm{C}$ for $15 \mathrm{sec}$ and $72^{\circ} \mathrm{C}$ for $45 \mathrm{sec}$ (40 cycles). Subsequently, the amplified product was subjected to melting curve analysis. Negative control reactions were conducted using the same amount of RNA without reverse transcription. Expression of each gene was normalized to GAPDH.

Preparation of isolated bladder cells. After sacrificing the rat, the whole bladder was aseptically isolated and washed in sterile phosphate-buffered saline (PBS) solution. Then, the urothelium was dissected away in the sterile $\mathrm{Ca}^{2+}$-free Hank's solution (Boster). The bladder was minced and incubated for $10 \mathrm{~min}$ at $37^{\circ} \mathrm{C}$ in $4 \mathrm{ml}$ enzyme solution. To terminate digestion, 4 ml RMPI-1640 medium (HyClone, Logan, UT, USA) containing $10 \%$ fetal bovine serum (FBS; Gibco, Life Technologies, Grand Island, NY, USA) was applied. The tissue suspension was blowed and the cells were collected using a 200-mesh cell strainer. The digestive process was repeated several times to harvest more cells. Finally, cells were plated onto sterile polylysin-coated glass coverslips in RMPI-1640 medium containing $10 \%$ FBS and $1 \%$ antibiotics/antimycotics (Beyotime Institute of Biotechnology), and cultured at $37^{\circ} \mathrm{C}$ in a $95 \% \mathrm{O}_{2}$ and $5 \% \mathrm{CO}_{2}$ incubator. The enzyme solution contained the following components (in $\mathrm{mg} / \mathrm{ml}$ ): 1.0 type II collagenase, 1.0 BSA and 1.0 trypsin inhibitor (all from Sigma-Aldrich, St. Louis, MO, USA).

Immunofluorescence. Cells were fixed in $4 \%$ paraformaldehyde (Boster) for $30 \mathrm{~min}$, and washed in PBS (10 min x 3 times). To block non-specific epitopes, cells were incubated with immunostaining blocking buffer (Beyotime Institute of Biotechnology) for $60 \mathrm{~min}$. Then cells were incubated overnight at $4^{\circ} \mathrm{C}$ with primary antibodies: c-kit (sc-1494, 1:50; Santa Cruz Biotechnology, Inc., Santa Cruz, CA, USA), HCN1 (ab84816, 1:100), HCN2 (ab65704, 1:100), HCN3 (ab84818, 1:100) and HCN4 (ab69054, 1:100) (all from Abcam), followed by the appropriate fluorescence-conjugated secondary antibodies: Alexa Fluor 488 mouse anti-goat IgG (bs-0294M, 1:200; Bioss,
Beijing, China), Alexa Fluor 647 goat anti-mouse IgG (P0191, 1:200) and Alexa Fluor 647 goat anti-rabbit IgG (P0180, 1:200) (both from Beyotime Institute of Biotechnology). Next, cells were incubated with 2-(4-amidinophenyl)-6-indolecarbamidine dihydrochloride (DAPI; Beyotime Institute of Biotechnology) to label the cell nucleus. Negative control was performed by omitting the primary antibody. All incubation steps were followed by washes with PBS (10 min x 3 times). Cells were visualized and photographed using a confocal laser scanning microscope (Leica, Wetzlar, Germany). The mean fluorescence density was measured by Image-Pro Plus version 6.0 software (Media Cybernetics, Inc., Rockville, MD, USA).

Patch-clamp. After regularly culturing for 1-2 days, the patch-clamp technique was performed on the bladder ICC-LCs, which were distinguished by distinctive cell morphology, stellate or spindle shapes with multiple branches (9). Electrodes were pulled by model P-97 Flaming/Brown micropipette puller (Sutter Instrument, Novato, CA, USA), and possessed resistance of 4-6 M $\Omega$. In the whole-cell voltage-clamp mode, the cells were held at $-60 \mathrm{mV}$ and followed by a stepped potential from -60 to $-120 \mathrm{mV}$ in $-10 \mathrm{mV}$ increments. Then, the cells were subjected a voltage jump to $-120 \mathrm{mV}$ to fully activate the $\mathrm{HCN}$ channels. $\mathrm{I}_{\mathrm{h}}$ was amplified and recorded by the HEKA EPC10 USB amplifier (HEKA Elektronik, Lambrecht, Germany) and filtered at a threshold frequency of $2.9 \mathrm{kHz}$. To calculate the current density, $I_{h}$ was normalized to cell capacitance. The elements of intracellular pipette solution were (in $\mathrm{mM}$ ): $130 \mathrm{~K}$ aspartate, $0.1 \mathrm{Na}_{2} \mathrm{GTP}, 5 \mathrm{Na}_{2} \mathrm{ATP}, 2 \mathrm{MgCl}_{2}, 5 \mathrm{CaCl}_{2}, 11$ EGTA, 10 HEPES, pH 7.2 with $\mathrm{KOH}$. The bath solution included (in $\mathrm{mM}$ ): $140 \mathrm{NaCl}, 1.2 \mathrm{MgCl}_{2}, 5.4 \mathrm{KCl}, 1.8 \mathrm{CaCl}_{2}, 10$ glucose, 5 HEPES, pH 7.4 with $\mathrm{NaOH}$. $\mathrm{BaCl}_{2}(1 \mathrm{mM})$ and $0.001 \mathrm{mM}$ TTX (Tocris Bioscience, Bristol, UK) were added into the bath solution to pharmacologically isolate the $\mathrm{I}_{\mathrm{h}}$. The specific inhibitor of HCN channels, ZD7288 (50 $\mu \mathrm{M}$; Sigma-Aldrich), was applied to further validate the specificity of $I_{h}$.

Measurement of intracellular calcium ion concentration $\left(\left[\mathrm{Ca}^{2+}\right]_{i}\right)$. The $\left[\mathrm{Ca}^{2+}\right]_{\mathrm{i}}$ measurement was tested on the bladder cells that were cultured for 1-2 days. The primary isolated bladder cells were washed with Hank's solution (Boster) for 
A
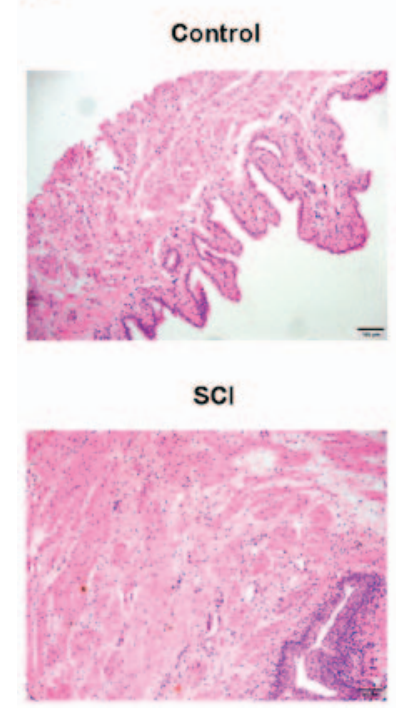
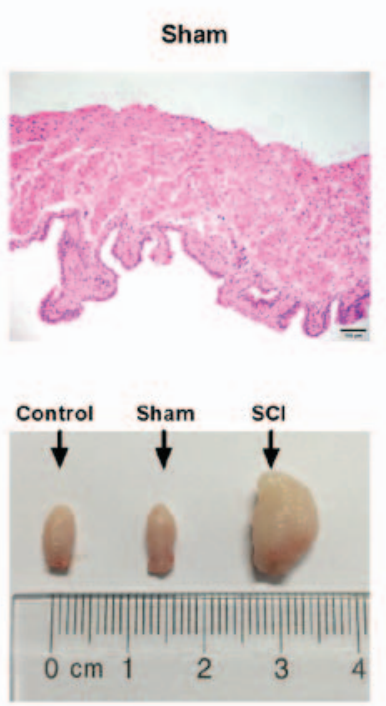

B
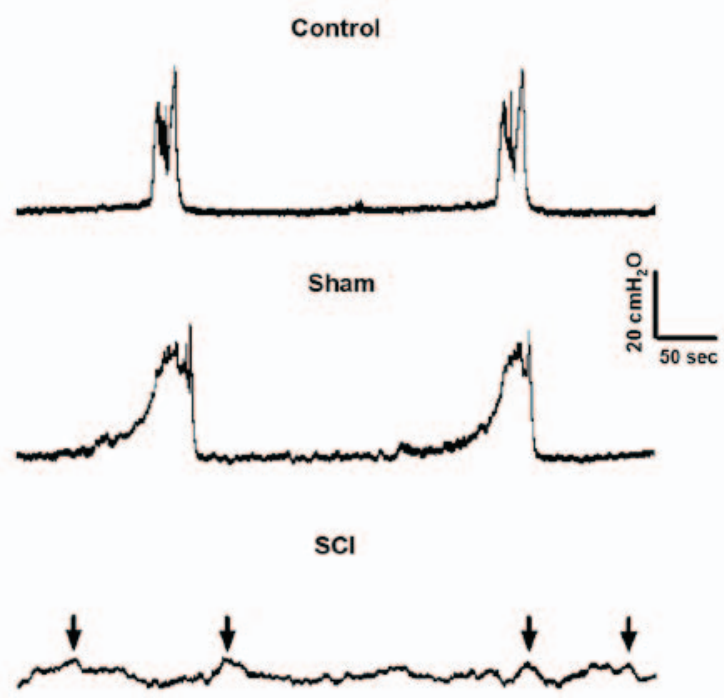

Figure 1. Assessment of spinal cord injury (SCI) rat models. (A) In bladder sections with hematoxylin and eosin (H\&E) staining, we observed the obviously hypertrophic muscle layer, incrassated urothelium and enriched mesenchyme matter in SCI rats under x100 magnification. Macroscopically, bladders in SCI rats were much greater than that in control and sham rats. (B) The regular voiding cycle of the bladder was detected in control and sham rats, while the common voiding contractions were replaced by several weaken and irregular micturition waves (black arrows) in SCI rats.

5 min and incubated with Fluo-4 AM $(10 \mu \mathrm{M}$; Molecular Probes, Eugene, OR, USA) for $30 \mathrm{~min}$ at $37^{\circ} \mathrm{C}$. Then, bladder cells were washed with Hank's solution (5 min x 3 times). $\mathrm{Ca}^{2+}$ imaging was performed on the bladder ICC-LCs using the laser scanning confocal microscope (Leica) at an emission wavelength of $488 \mathrm{~nm}$. Forskolin (FSK) $(50 \mu \mathrm{M}$; Sigma-Aldrich) and 8-bromoadenosine 3',5'-cyclic monophosphate (8-Br-cAMP) $(50 \mu \mathrm{M}$; Tocris Bioscience) were administered into the vessel, respectively. ZD7288 (50 $\mu \mathrm{M}$; Sigma-Aldrich) was applied to further confirm the reliability of isolated bladder ICC-LCs. The results were presented as the relative fluorescence intensity $(\mathrm{RFI}=\mathrm{F} 1 / \mathrm{F} 0$, where $\mathrm{F} 0$ is the baseline fluorescence intensity, and F1 is the real-time fluorescence intensity after drug administration).

Contractility studies. The dissected rat bladders were softly stripped, the urothelium layer was then longitudinally sheared into $\sim 3 \times 3 \times 8 \mathrm{~mm}$ strips. The strips were settled into a tissue bath filled with $15 \mathrm{ml}$ aerated Kreb's solution $\left(95 \% \mathrm{O}_{2}\right.$ and $5 \%$ $\mathrm{CO}_{2}$ at $37^{\circ} \mathrm{C}$ ), and suspended perpendicularly between two crooked hooks connected to the stretch transducer (Chengyi Co.) and fixed on the bottom of the bath, respectively. Stretch transducer was calibrated before every experiment. The strip was equilibrated for $30 \mathrm{~min}$ and stretched with $0.75 \mathrm{~g}$ tension. Two doses of the ZD7288 (10 and $50 \mu \mathrm{M}$; Sigma-Aldrich) were orderly added into the bathing solution at 6-min intervals. The continuous contraction curves were visualized with the RM6240C (Chengyi Co.). The Kreb's solution contained the following components (in $\mathrm{mM}$ ): $119 \mathrm{NaCl}, 4.7 \mathrm{KCl}$, $1.2 \mathrm{KH}_{2} \mathrm{PO}_{4}, 1.2 \mathrm{MgSO}_{4} \cdot 7 \mathrm{H}_{2} \mathrm{O}, 25 \mathrm{NaHCO}_{3}, 2.5 \mathrm{CaCl}_{2}$ and 11 glucose, adjusted to $\mathrm{pH} 7.2$ with $\mathrm{NaOH}$.

Statistical analysis. All experimental data are presented as mean \pm SD. Significance between each group was analyzed using one-way analysis of variance (ANOVA) in
SPSS version 13.0 software (SPSS Inc., Chicago, IL, USA). P-values $<0.05$ were regarded as significant. All experiments were conducted with a minimum of three independent replications.

\section{Results}

Changed morphology and histology of bladder after SCI. The changes of bladder histology after SCI were assessed by H\&E staining. Under x100 magnification, we found that the muscle layers manifested obvious hypertrophy and urothelium were noticeably incrassated, accompanied by abundant mesenchyme matter in SCI rats compared with control and sham rats. Macroscopically, bladders in SCI rats were much greater than that in control and sham rats (Fig. 1A).

SCI rats exhibited remarkable urodynamics changes. As shown in Fig. 1B, in control and sham rats, we detected the disciplinary voiding cycle or contractility of the bladder. However, we did not observe common voiding contractions of the bladder in SCI rats, which were replaced by several irregular micturition waves with low amplitude (black arrows).

Decreased mRNA and protein expression levels of HCN channels in SCI rat bladders. Using quantitative RT-PCR and western blot analysis, we detected the changes in $\mathrm{HCN}$ channel expression in SCI rat bladders. The mRNA and protein expression levels of four HCN subtypes were significantly decreased in SCI rat bladders, and the alteration of the HCN1 channel was the most significant (Fig. 2A, B and D). To test the HCN channel expression levels in single bladder ICC-LC, we utilized immunofluorescent staining. The results revealed that all four HCN subtypes were expressed in single bladder ICC-LC which was labeled by c-kit primary antibody, but the mean fluorescence density of four HCN subtypes was significantly 
A

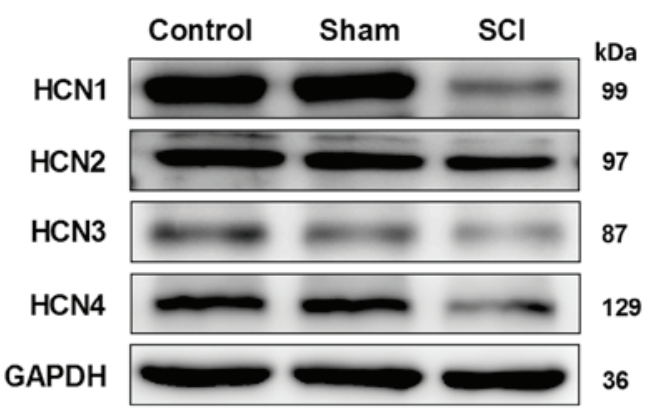

B

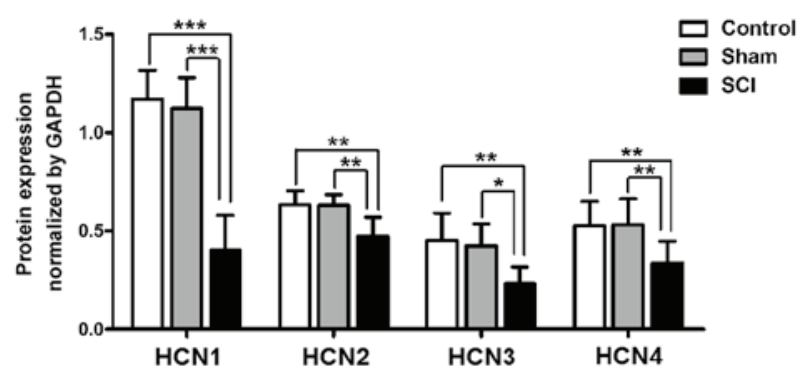

C
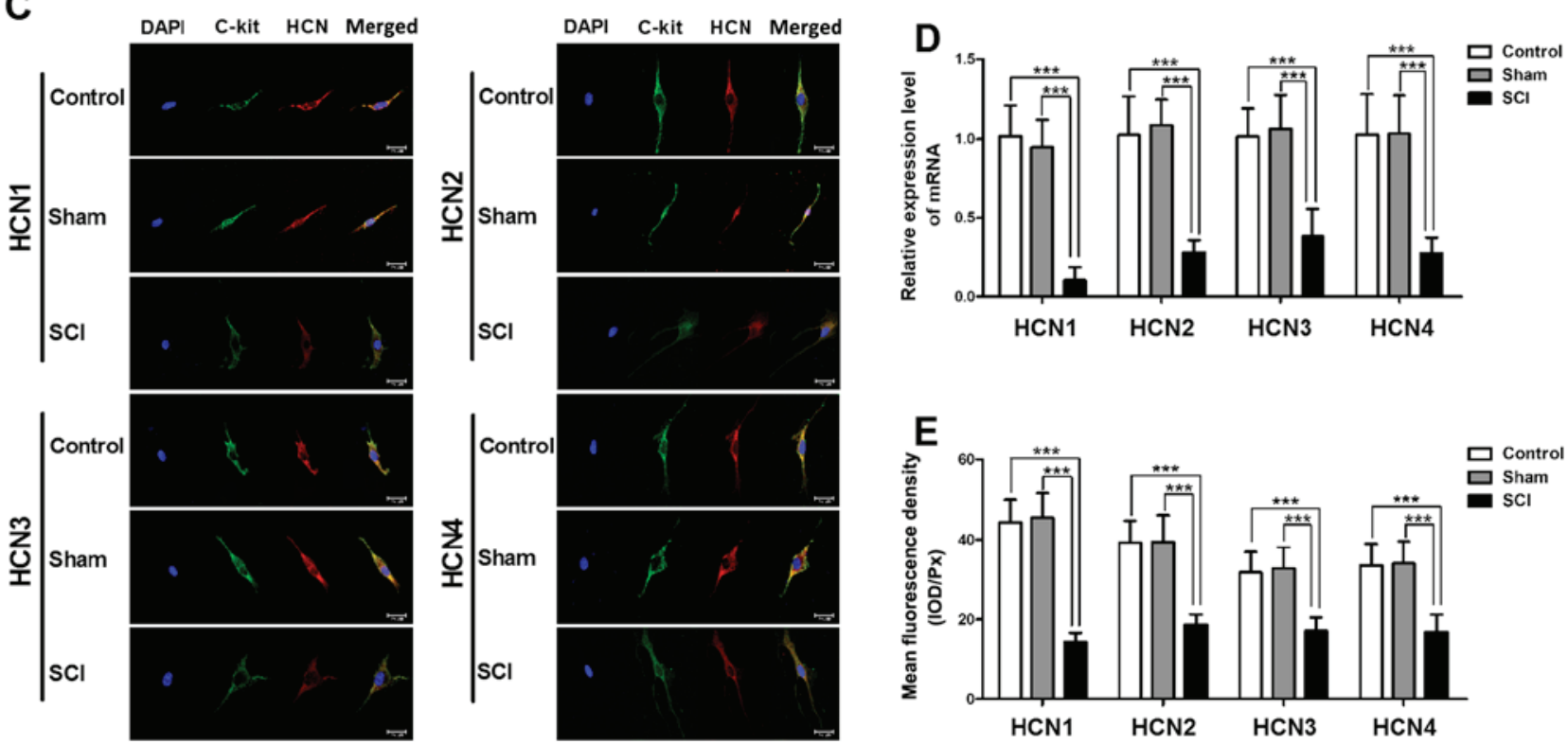

Figure 2. Reduced mRNA and protein expression levels of hyperpolarization-activated cyclic nucleotide-gated (HCN) channels in bladder interstitial cells of Cajal-like cells (ICC-LCs) after spinal cord injury (SCI). (A, B and D) The mRNA and protein expression levels of four HCN subtypes in bladder were significantly decreased after SCI, and the decrease of $\mathrm{HCN} 1$ channel was the most significant $\left({ }^{*} \mathrm{P}<0.05,{ }^{* * *} \mathrm{P}<0.01\right.$ and $\left.{ }^{* * * *} \mathrm{P}<0.001\right)$. (C) Using immunofluorescence staining, we found that four HCN subtypes (red) were expressed in bladder ICC-LCs that were labeled by C-kit (green) in all three groups. (E) The expression levels of HCN channels in single bladder ICC-LC are represented by mean fluorescence density. The expression levels of all four HCN subtypes exhibited significant decrease in SCI rats and the HCN1 channel expression was also the most significantly altered one $\left({ }^{* * *} \mathrm{P}<0.001\right)$.

decreased in SCI rat bladders and the HCN1 channel also exhibited most significant decrease (Fig. 2C and E).

Changes in $I_{h}$ properties in bladder ICC-LCs after SCI. To evaluate whether the $\mathrm{HCN}$ channel function was changed after SCI, we utilized the whole-cell patch clamp to record $\mathrm{I}_{\mathrm{h}}$ in isolated bladder ICC-LCs. As shown in Fig. 3A, $\mathrm{I}_{\mathrm{h}}$ was recorded in all three groups. Moreover, the specificity of these recorded $\mathrm{I}_{\mathrm{h}}$ was validated by ZD7288 that reduced the $\mathrm{I}_{\mathrm{h}}$ amplitudes. Fig. 3B shows that $I_{h}$ amplitude and HCN channel gating rate in SCI rats were significantly decreased at the potential of $-120 \mathrm{mV}$. When normalized to cell capacitance, $\mathrm{I}_{\mathrm{h}}$ density in SCI rats was significantly decreased compared with that in control and sham rats over the voltage range of -60 to $-120 \mathrm{mV}$, and the decrease of $\mathrm{I}_{\mathrm{h}}$ density at the potential of $-120 \mathrm{mV}$ in SCI rats was the most significant (Fig. 3C and D).

Altered expression levels of HCN1 channel regulatory proteins in SCI rat bladders. To investigate whether the expression levels of HCN1 channel regulatory proteins were changed in SCI rat bladders, we detected the protein expression of Trip8b, filamin A, Nedd4-2 and NRSF. We found that the protein expression levels of Trip8b, Nedd4-2 and NRSF were significantly upregulated, while the protein expression level of filamin A was significantly downregulated in SCI rat bladders (Fig. 4).

Decreased bladder ICC-LC excitability in SCI rats. We conducted $\left(\left[\mathrm{Ca}^{2+}\right]_{\mathrm{i}}\right)$ measurements to estimate the bladder ICC-LC excitability in each group. Upon the application of FSK $(50 \mu \mathrm{M})$ and 8 -Br-cAMP $(50 \mu \mathrm{M}),\left[\mathrm{Ca}^{2+}\right]_{\mathrm{i}}$ in isolated bladder ICC-LCs from all three groups was significantly elevated. Furthermore, the increased $\left[\mathrm{Ca}^{2+}\right]_{\mathrm{i}}$ in isolated bladder ICC-LCs could be reversed by the ZD7288 $(50 \mu \mathrm{M})$ administration (Fig. 5A and B). Therefore, the authenticity of isolated bladder ICC-LCs which were chosen for testing by morphology was further confirmed by this consequence. The enhancements of $\left[\mathrm{Ca}^{2+}\right]_{\mathrm{i}}$ facilitated by FSK or 8-Br-cAMP in SCI rats were significantly weaker than that in control and sham rats (Fig. 5C-F).

Decreased tolerance of detrusor strip to ZD7288 in SCI rats. In the contractility studies, isolated detrusor strips from all three groups generated spontaneous contractions. The 

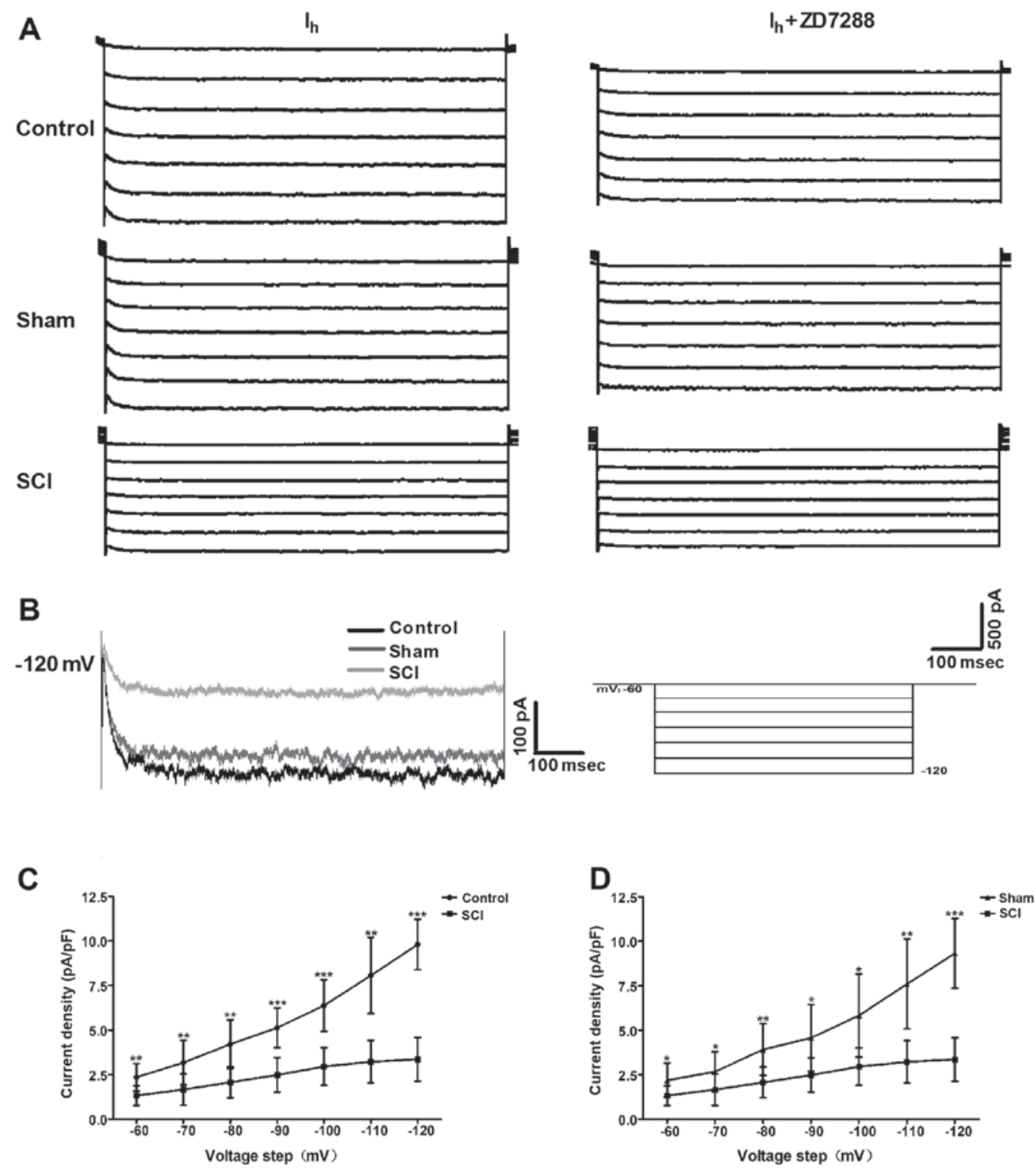

Figure 3. Alterations in $I_{h}$ properties of bladder interstitial cells of Cajal-like cells (ICC-LCs) from spinal cord injury (SCI) rats. (A) Upon a voltage-clamp protocol with a holding potential of $-60 \mathrm{mV}$ and a stepped potential from -60 to $-120 \mathrm{mV}$ in increments of $-10 \mathrm{mV}$, characteristic $\mathrm{I}_{\mathrm{h}}$ was recorded in bladder ICC-LCs from all three groups. ZD7288 (50 $\mu \mathrm{M})$ significantly reduced the $\mathrm{I}_{\mathrm{h}}$ amplitude in all three groups, which confirmed the specificity of these recorded $I_{h}$. (B) When HCN channels were fully activated at the potential of $-120 \mathrm{mV}$, the $I_{b}$ amplitude and the HCN channel gating rate were significantly declined in SCI rats. (C and D) Compared with that in control and sham rats, the $\mathrm{I}_{\mathrm{h}}$ density normalized to cell capacitance was significantly decreased in SCI rats, over the voltage range of -60 to $-120 \mathrm{mV}\left({ }^{*} \mathrm{P}<0.05,{ }^{* *} \mathrm{P}<0.01\right.$ and $\left.{ }^{* * *} \mathrm{P}<0.001\right)$.

contraction amplitude and frequency of detrusor strips were significantly reduced in SCI rats (Fig. 6A). The low ZD7288 $(10 \mu \mathrm{M})$ concentration did not influence the phasic contractions of detrusor strips in control and sham groups, but significantly decreased the contraction amplitude (Fig. 6B), and increased the frequency of detrusor strips in SCI rats (Fig. 6C). Only at the high ZD7288 $(50 \mu \mathrm{M})$ concentration, the phasic contractions of detrusor strips in all three groups were significantly inhibited (Fig. 6).

\section{Discussion}

In the present study, we demonstrated that the HCN channel expression and function were significantly reduced in rats with SCI induced neurogenic bladder, and the expression levels of several HCN1 channel regulatory proteins were also significantly altered. Then, we found that the bladder ICC-LC excitability and detrusor strip contractility were significantly declined due to the decrease of HCN channels. These findings indicate that decreased $\mathrm{HCN}$ channels and impaired bladder ICC-LCs are involved in the pathogenesis of SCI induced neurogenic bladder.

As we know, the primary micturition center locates in the sacral spinal cord level S2-S4 and damage at this level commonly leads to a highly compliant acontractile bladder (21). This is supported by our results detected in histomorphology test and urodynamic measurement. Moreover, we found that although the regular voiding contraction was disappeared in SCI rat bladders, several weaken and irregular micturition waves were existed. This finding prompts us that the bladder possess the capacity of spontaneous excitement to modulate bladder contraction under the condition that lost disciplinary 

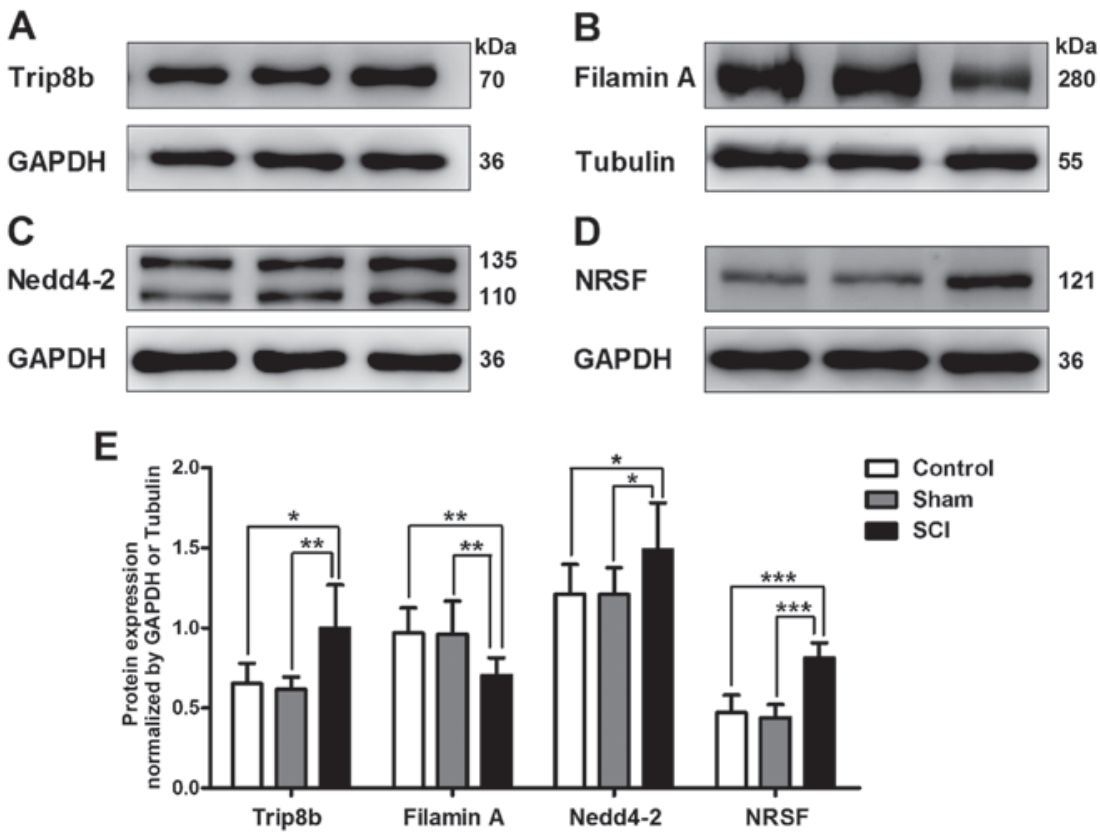

Figure 4. Changed expression levels of hyperpolarization-activated cyclic nucleotide-gated (HCN)1 channel regulatory proteins in spinal cord injury (SCI) rat bladders. Using western blot analysis, the protein expression level was detected of (A) tetratricopeptide repeat-containing Rab8b-interacting protein (Trip8b), (B) filamin A, (C) neural precursor cell-expressed developmentally downregulated (Nedd)4-2 and (D) neuronal restrictive silencing factor (NRSF) in bladders of all three groups. (E) Compared with that in control and sham rat bladders, the protein expression levels of Trip8b, Nedd4-2 and NRSF were significantly increased, while the protein expression level of filamin A was significantly downregulated in SCI rat bladders ( $\mathrm{P}<0.05,{ }^{* * *} \mathrm{P}<0.01$ and $\left.{ }^{* * * *} \mathrm{P}<0.001\right)$.
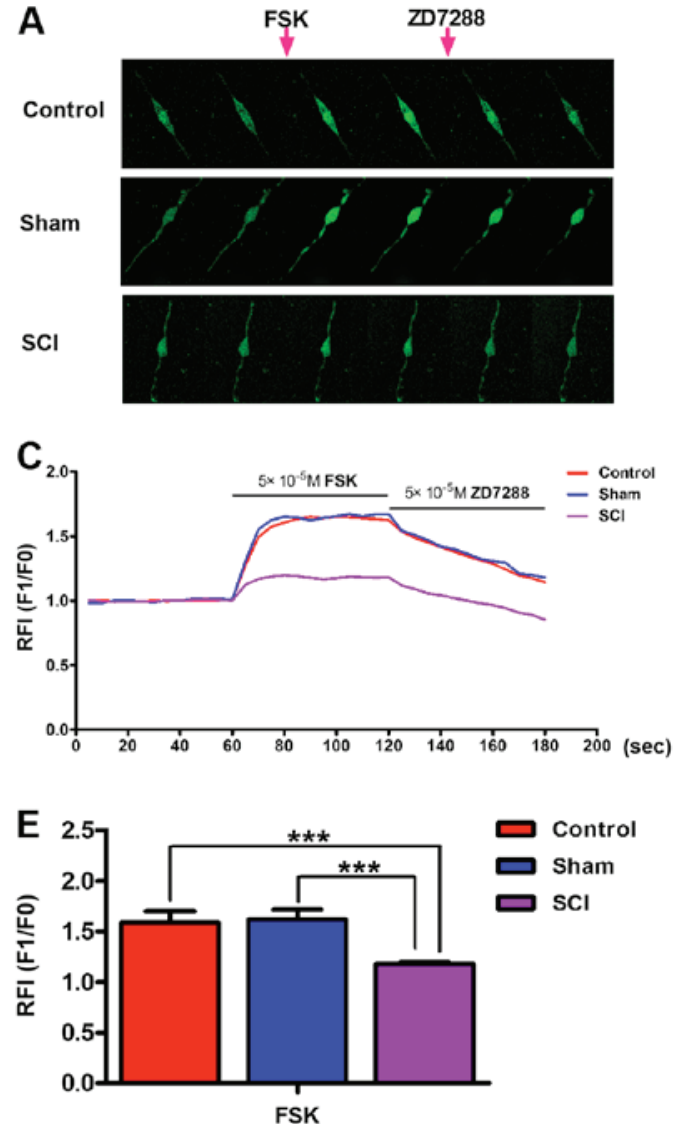
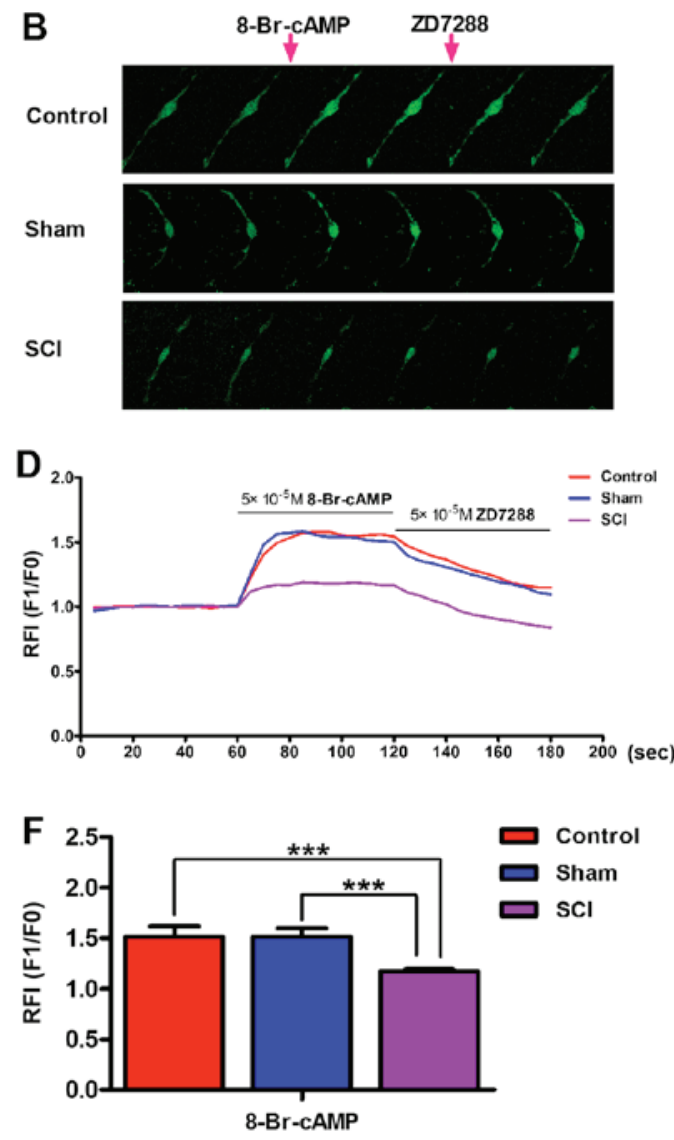

Figure 5. Effects of forskolin (FSK) and 8-bromoadenosine 3',5'-cyclic monophosphate (8-Br-cAMP) on the intracellular calcium ion concentration $\left(\left[\mathrm{Ca}^{2+}\right]\right)$ in bladder interstitial cells of Cajal-like cells (ICC-LCs). (A and B) $\left[\mathrm{Ca}^{2+}\right]_{\mathrm{i}}$ measurements were performed in isolated bladder ICC-LCs. Six images were selected in each group to present continuous alterations of real-time $\left[\mathrm{Ca}^{2+}\right]_{i}$ upon the administration of drugs (FSK, 8-Br-cAMP, ZD7288). FSK or 8-Br-cAMP and ZD7288 were applied after recording for 1 and $2 \mathrm{~min}$, respectively. (C and D) The impacts of the three pharmaceuticals on the $\left[\mathrm{Ca}^{2+}\right]_{\mathrm{i}}$ in each group were represented as the relative fluorescence intensities (RFI, F1/F0) and illustrated as continuous curves. FSK $(50 \mu \mathrm{M})$ and 8 -Br-cAMP $(50 \mu \mathrm{M})$ both significantly elevated the $\left[\mathrm{Ca}^{2+}\right]_{\mathrm{i}}$, while ZD7288 $(50 \mu \mathrm{M})$ decreased the $\left[\mathrm{Ca}^{2+}\right]_{\mathrm{i}}$ in all three groups. The effects of (E) FSK or (F) 8 -Br-cAMP on the $\left[\mathrm{Ca}{ }^{2+}\right]_{\mathrm{i}}$ in bladder ICC-LCs from spinal cord injury (SCI) rats were significantly decreased $\left({ }^{* * * *} \mathrm{P}<0.001\right)$. 
A

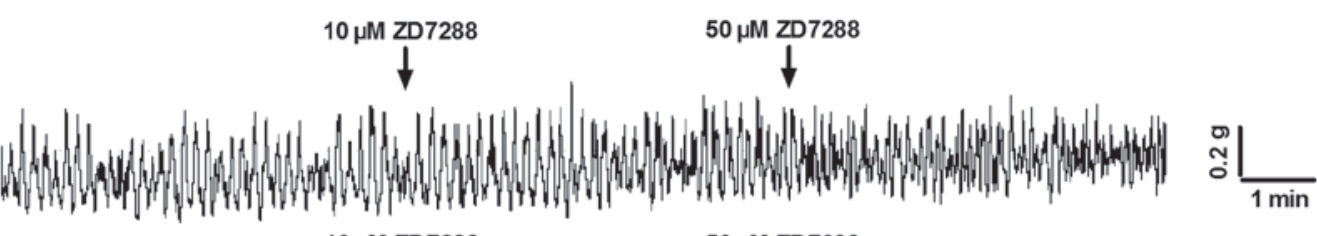

Control

$10 \mu \mathrm{M}$ ZD7288

$50 \mu \mathrm{M} Z \mathrm{ZD} 7288$

$\downarrow$

$\downarrow$

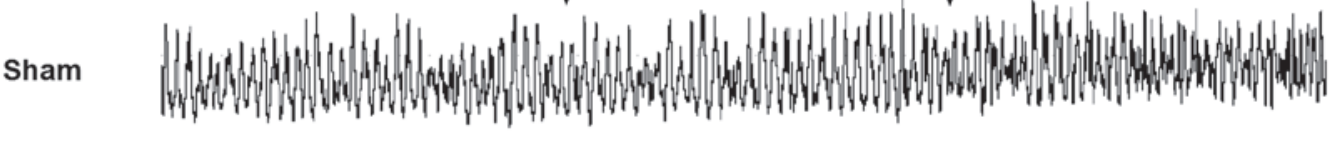

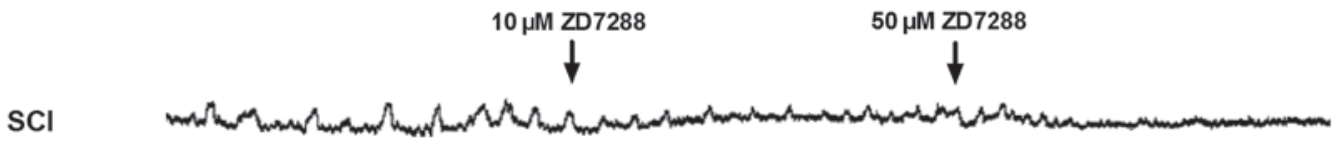

B

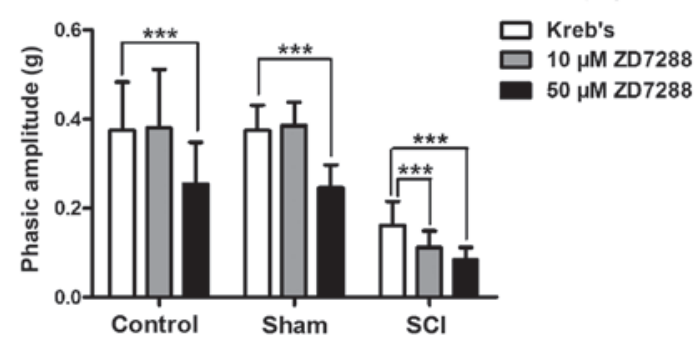

C

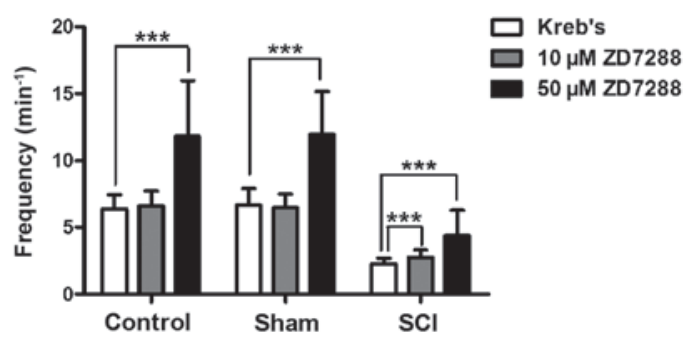

Figure 6. Altered spontaneous contractions and responses to ZD7288 of detrusor strips in SCI rats. (A) All isolated detrusor strips generated spontaneous contractions. Detrusor strips in spinal cord injury (SCI) rats exhibited significantly declined amplitude and frequency of spontaneous contractions. Detrusor strips in each group showed different responses to two doses of ZD7288 (10 and $50 \mu \mathrm{M}$ ). (B and C) ZD7288 (10 $\mu \mathrm{M})$ did not affect the phasic contractions of detrusor strips in control and sham rats, but significantly decreased the contraction amplitude and enhanced the frequency of detrusor strip in SCI rats. Only $50 \mu \mathrm{M}$ ZD7288 can significantly suppress the phasic contractions of detrusor strips in all three groups $\left({ }^{* * *} \mathrm{P}<0.001\right)$.

neural control. Thus, in this study, we focused on the investigation of the changes of spontaneous excitability in SCI induced neurogenic bladder.

HCN channels which can generate the pacemaker current, $I_{h}$, are deemed to play an important role in the bladder pacemaker activity (13). In our study, we demonstrated that the gene and protein expression levels of four $\mathrm{HCN}$ subtypes were significantly decreased in SCI induced neurogenic bladder. Given that bladder ICC-LC quantities were significantly decreased in rat with sacral spinal cord (17), therefore, we investigated whether the HCN channel expression levels in individual bladder ICC-LC were changed. To solve our confusion, we conducted immunofluorescence staining, in which we observed that the protein expression levels of HCN channels in single bladder ICC-LC was also signicantly reduced. Furthermore, these data were supported by the results we detected in patch-clamp experiments that the $\mathrm{I}_{\mathrm{h}}$ density was significantly decreased in individual ICC-LC of SCI induced neurogenic bladder. The HCN channel gating rate was obviously decelerated after SCI. In conclusion, we suggest that the decreased $\mathrm{HCN}$ channel expression and function are associated with the pathogenesis of SCI induced neurogenic bladder.

In addition, we validated that the HCN1 channel exhibited the most significant alterations in gene and protein expression levels after SCI, highlighting the crucial role of HCN1 channels in SCI induced bladder dysfunction. Hence, we mainly explored whether the expression levels of HCN1 channel regulatory proteins were changed in SCI induced neurogenic bladder. As we expected, we found that the expression levels of four HCN1 channel regulatory proteins including Trip8b, filamin A, Nedd4-2 and NRSF in bladder were all significantly altered after SCI. We ascribe the significant reduction of $\mathrm{HCN} 1$ channel expression to the significantly increased transcription factor, NRSF, which can bind to the HCN1 gene promoter and reduce the HCN1 channel gene transcription (22). Furthermore, Trip8b, which contains abundant alternative $\mathrm{N}$-terminal splice variants, is an important regulatory subunit of $\mathrm{HCN} 1$ channel in the mammalian brain. Different Trip8b splice variants generate various effects on $\mathrm{HCN} 1$ channel membrane trafficking, while all splice isoforms suppress $\mathrm{HCN} 1$ channel gating by antagonizing the function of cyclic nucleotides (23). The cytoplasmic scaffolding protein filamin A can interact with the C-terminal of $\mathrm{HCN} 1$ channel through a 22-amino acid region and enhance HCN1 channel surface expression (24). In contrast, the Nedd4-2 interacts with the $\mathrm{C}$-terminus of $\mathrm{HCN} 1$ channel, then decreases its surface expression and negatively modulates the channel gating (25). Taken together, we speculate that the decrease in $\mathrm{I}_{\mathrm{h}}$ density and $\mathrm{HCN}$ channel gating rate in SCI rat bladders may be largely attributed to the alterations in $\mathrm{HCN} 1$ channel regulatory proteins. Moreover, we believe that the altered regulatory proteins of HCN1 channel and even of other $\mathrm{HCN}$ subtypes play an important role in SCI induced neurogenic bladder.

To our knowledge, the functional role of $\mathrm{HCN}$ channels in regulating cytomembrane electrophysiology has been well clarified. HCN channels can be activated by membrane hyperpolarization or cyclic nucleotides, particularly cyclic adenosine 
monophosphate (cAMP), and depolarize membrane potential toward the threshold for firing action potential by generating an inward current, $\mathrm{I}_{\mathrm{h}}$ (26). Moreover, such HCN channels induced pacemaker depolarization subsequently activate the low-voltage-activated $\mathrm{Ca}^{2+}$ channels, such as T-type or L-type $\mathrm{Ca}^{2+}$ channels, and facilitate robust $\mathrm{Ca}^{2+}$ influx into cells (27). Therefore, HCN channels may not only act as pacemaker channels to initiate rhythmic burst firing in neurons and to control cardiac rhythm (28), but also regulate the spontaneous pacemaker potentials in cultured colonic ICCs (29). In this study, we performed $\left[\mathrm{Ca}^{2+}\right]_{\mathrm{i}}$ measurements to detect the effects of HCN channels on the excitability of bladder ICC-LCs, which also possess the ability to generate spontaneous action potential (30). Upon the administration of the two HCN channel agonists (FSK and 8-Br-cAMP), prominent $\mathrm{Ca}^{2+}$ influx was observed in bladder ICC-LCs from normal and sham rats, indicating that $\mathrm{HCN}$ channels may also participate in modulating the bladder ICC-LC pacemaker activity. However, the effects of FSK and 8-Br-cAMP on the bladder ICC-LCs in SCI group were significantly weakened, further highlighting the important role of HCN channels in regulating bladder ICC-LC excitability. Furthermore, we suggest that the impaired bladder ICC-LCs ascribed to decreased HCN channel expression and function are involved in SCI induced neurogenic bladder.

Bladder ICC-LCs are deemed to control the spontaneous contractions of detrusor smooth muscle (31). Thus, in our study, corresponding to the decreased bladder ICC-LC excitability, we found that detrusor strips in SCI rats generated much weaker spontaneous phasic contractions than that in normal and sham rats. We further detected that the tolerance of detrusor strips to ZD7288 were decreased in SCI rats. These results could also be attributed to the decreased $\mathrm{HCN}$ channel expression and function. Accordingly, we conclude that $\mathrm{HCN}$ channels can control the spontaneous contraction of detrusor strips via regulating bladder ICC-LC excitability, and correspondingly, altered $\mathrm{HCN}$ channel expression and function in pathologic conditions may lead to disordered detrusor strip contractility by damaging the bladder ICC-LC function.

Compounds targeting $\mathrm{HCN}$ channels are not only widely utilized to treat several heart diseases and neuropathic pain, but also used as anticonvulsant and anaesthetic drugs (32). For example, ivabradine is the first clinically approved medication that specifically targets $\mathrm{HCN}$ channels to treat chronic stable angina pectoris in patients with contraindication or intolerance for $\beta$-blockers (33). In the present study, our data indicate that HCN channels in bladder ICC-LCs may be considered as a novel therapeutic target to improve bladder function in SCI induced neurogenic bladder. The limitation of our study is that we only tested the functional role of bladder HCN channels in rats subjected with sacral cord injury. To more completely characterize the role of $\mathrm{HCN}$ channels in SCI induced neurogenic bladder, animal models with suprasacral cord injury should be used in further studies.

In conclusion, our findings illustrate the possible mechanism that decreases in $\mathrm{HCN}$ channel expression and function induced by altered regulatory protein expression, can impair the bladder ICC-LC excitability and damage the detrusor strip contractility, and then lead to bladder dysfunction. The present mechanism may be involved in the pathological process of SCI induced neurogenic bladder.

\section{Acknowledgements}

Not applicable.

\section{Funding}

The present study was supported by the National Natural Science Foundation of China (NSFC) (grant nos. 81230017 and 81300630).

\section{Availability of data and material}

The datasets used and/or analyzed during the current study are available from the corresponding author on reasonable request.

\section{Authors' contributions}

QL performed the major experiments and drafted the manuscript. CW and SH analyzed the data. QW and TZ participated in the hematoxylin and eosin staining, western blot analyses, quantitative RT-PCR and immunofluorescence. XL, XL and XH were involved in the patch-clamp experiments, $\left[\mathrm{Ca}^{2+}\right]_{\mathrm{i}}$ measurements, contractility studies and urodynamic measurements. LL was responsible for designing the experiments and editing the paper. All authors read and approved the final manuscript.

\section{Ethics approval and consent to participate}

All animal experiments were performed according to the Guide for Care and Use of Laboratory Animals issued by the National Institutes of Health, and were authorized by the Research Council and Animal Care and Use Committee of the Third Military Medical University, China.(approval no. SYXK20070002).

\section{Consent for publication}

Not applicable.

\section{Competing interests}

The authors declare that they have no competing interests.

\section{References}

1. Thuret S, Moon LD and Gage FH: Therapeutic interventions after spinal cord injury. Nat Rev Neurosci 7: 628-643, 2006.

2. Kim JH, Shim SR, Doo SW, Yang WJ, Yoo BW, Kim JM, Ko YM, Song ES, Lim IS, Lee HJ, et al: Bladder recovery by stem cell based cell therapy in the bladder dysfunction induced by spinal cord injury: systematic review and meta-analysis. PLoS One 10: e0113491, 2015.

3. $\mathrm{Ku} \mathrm{JH}$ : The management of neurogenic bladder and quality of life in spinal cord injury. BJU Int 98: 739-745, 2006.

4. Cetinel B, Onal B, Can G, Talat Z, Erhan B and Gunduz B: Risk factors predicting upper urinary tract deterioration in patients with spinal cord injury: a retrospective study. Neurourol Urodyn 36: 653-658, 2017.

5. Harris CJ and Lemack GE: Neurourologic dysfunction: evaluation, surveillance and therapy. Curr Opin Urol 26: 290-294, 2016

6. Zhang T, Liu H, Liu Z and Wang L: Acupuncture for neurogenic bladder due to spinal cord injury: a systematic review protocol. BMJ Open 4: e006249, 2014. 
7. Gfroerer S and Rolle U: Interstitial cells of Cajal in the normal human gut and in Hirschsprung disease. Pediatr Surg Int 29: 889-897, 2013.

8. Sanders KM, Ward SM and Koh SD: Interstitial cells: regulators of smooth muscle function. Physiol Rev 94: 859-907, 2014.

9. McCloskey KD: Interstitial cells in the urinary bladder - localization and function. Neurourol Urodyn 29: 82-87, 2010.

10. McCloskey KD: Bladder interstitial cells: an updated review of current knowledge. Acta Physiol (Oxf) 207: 7-15, 2013.

11. Deng J, Zhang Y, Wang L, Zhao J, Song B and Li L: The effects of Glivec on the urinary bladder excitation of rats with suprasacral or sacral spinal cord transection. J Surg Res 183: 598-605, 2013.

12. Yang Q, Kuzyk P, Antonov I, Bostwick CJ, Kohn AB, Moroz LL and Hawkins RD: Hyperpolarization-activated, cyclic nucleotide-gated cation channels in Aplysia: contribution to classical conditioning. Proc Natl Acad Sci USA 112: 16030-16035, 2015.

13. He P, Deng J, Zhong X, Zhou Z, Song B and Li L: Identification of a hyperpolarization-activated cyclic nucleotide-gated channel and its subtypes in the urinary bladder of the rat. Urology 79 1411.e7-1411.e13, 2012.

14. He C, Chen F, Li B and Hu Z: Neurophysiology of HCN channels: from cellular functions to multiple regulations. Prog Neurobiol 112: 1-23, 2014.

15. Sartiani L, Romanelli MN, Mugelli A and Cerbai E: Updates on $\mathrm{HCN}$ channels in the heart: function, dysfunction and pharmacology. Curr Drug Targets 16: 868-876, 2015.

16. O'Donnell AM, Coyle D and Puri P: Decreased expression of hyperpolarisation-activated cyclic nucleotide-gated channel 3 in Hirschsprung's disease. World J Gastroenterol 21: 5635-5640, 2015.

17. Deng T, Zhang Q, Wang Q, Zhong X and Li L: Changes in hyperpolarization-activated cyclic nucleotide-gated channel expression and activity in bladder interstitial cells of Cajal from rats with detrusor overactivity. Int Urogynecol J Pelvic Floor Dysfunct 26: 1139-1145, 2015.

18. Lucin KM, Sanders VM, Jones TB, Malarkey WB and Popovich PG: Impaired antibody synthesis after spinal cord injury is level dependent and is due to sympathetic nervous system dysregulation. Exp Neurol 207: 75-84, 2007.

19. Fischer AH, Jacobson KA, Rose J and Zeller R: Hematoxylin and eosin staining of tissue and cell sections. CSH Protoc: pdb.prot4986, 2008. doi: 10.1101/pdb.prot4986.

20. Andersson KE, Soler R and Füllhase C: Rodent models for urodynamic investigation. Neurourol Urodyn 30: 636-646, 2011.
21. Goldmark E, Niver B and Ginsberg DA: Neurogenic bladder: from diagnosis to management. Curr Urol Rep 15: 448, 2014.

22. Bender RA and Baram TZ: Hyperpolarization activated cyclic-nucleotide gated $(\mathrm{HCN})$ channels in developing neuronal networks. Prog Neurobiol 86: 129-140, 2008.

23. Santoro B, Piskorowski RA, Pian P, Hu L, Liu H and Siegelbaum SA: TRIP8b splice variants form a family of auxiliary subunits that regulate gating and trafficking of $\mathrm{HCN}$ channels in the brain. Neuron 62: 802-813, 2009.

24. Gravante B, Barbuti A, Milanesi R, Zappi I, Viscomi C and DiFrancesco D: Interaction of the pacemaker channel HCN1 with filamin A. J Biol Chem 279: 43847-43853, 2004.

25. Wilkars W, Wollberg J, Mohr E, Han M, Chetkovich DM, Bähring R and Bender RA: Nedd4-2 regulates surface expression and may affect $N$-glycosylation of hyperpolarization-activated cyclic nucleotide-gated (HCN)-1 channels. FASEB J 28: 2177-2190, 2014.

26. Biel M, Wahl-Schott C, Michalakis S and Zong X: Hyperpolarization-activated cation channels: from genes to function. Physiol Rev 89: 847-885, 2009.

27. Robinson RB and Siegelbaum SA: Hyperpolarization-activated cation currents: from molecules to physiological function. Annu Rev Physiol 65: 453-480, 2003.

28. Benarroch EE: HCN channels: function and clinical implications. Neurology 80: 304-310, 2013.

29. Shahi PK, Choi S, Zuo DC, Kim MY, Park CG, Kim YD, Lee J, Park KJ, So I and Jun JY: The possible roles of hyperpolarization-activated cyclic nucleotide channels in regulating pacemaker activity in colonic interstitial cells of Cajal. J Gastroenterol 49: 1001-1010, 2014.

30. Wu Y, Shi C, Deng J, Zhang X, Song B and Li L: Expression and function of muscarinic subtype receptors in bladder interstitial cells of cajal in rats. Urol J 11: 1642-1647, 2014.

31. Kubota Y, Biers SM, Kohri K and Brading AF: Effects of imatinib mesylate (Glivec) as a c-kit tyrosine kinase inhibitor in the guinea-pig urinary bladder. Neurourol Urodyn 25: 205-210, 2006.

32. Postea $\mathrm{O}$ and Biel M: Exploring $\mathrm{HCN}$ channels as novel drug targets. Nat Rev Drug Discov 10: 903-914, 2011.

33. Riccioni G, Vitulano N and D'Orazio N: Ivabradine: beyond heart rate control. Adv Ther 26: 12-24, 2009. International (CC BY-NC-ND 4.0) License. 